\title{
Lateral approach improves surgical access to the superior pole in giant endemic goiters
}

\author{
Carolyn DeBiase ${ }^{1}$, Merry Sebelik $^{2}$, Srinivasa Rama Chandra ${ }^{3}$, Jagdish Dhingra ${ }^{4}$ \\ ${ }^{1}$ Department of Otolaryngology, Mayo Clinic, Phoenix, Arizona, USA; ${ }^{2}$ Department of Otolaryngology Head and Neck Surgery, Emory University \\ School of Medicine, Atlanta, Georgia, USA; ${ }^{3}$ Division of Oral and Maxillofacial Surgery, Head and Neck Surgery, University of Nebraska Medical \\ Center, Omaha, Nebraska, USA; ${ }^{4}$ Department of Otolaryngology Head and Neck Surgery, Tufts University Medical Center, Boston, Massachusetts, \\ USA \\ Contributions: (I) Conception and design: J Dhingra, M Sebelik, SR Chandra; (II) Administrative support: J Dhingra, M Sebelik; (III) Provision of \\ study materials or patients: J Dhingra, M Sebelik; (IV) Collection and assembly of data: J Dhingra, M Sebelik, C DeBiase; (V) Data analysis and \\ interpretation: All authors; (VI) Manuscript writing: All authors; (VII) Final approval of manuscript: All authors. \\ Correspondence to: Jagdish Dhingra, MD. 35 Pearl Street, 1st Floor, Brockton, MA 02301, USA. Email: jdhingra@entspecialists.com.
}

\begin{abstract}
Background: Surgical management of mega-goiters in endemic areas with extreme iodine deficiency presents unique challenges. Based on our initial 5-year experience (2007 to 2011) operating on mega-goiters in Gitwe, Rwanda, Africa, we modified our technique to a lateral approach which affords better exposure of the superior pole vessels and other vital neurovascular structures, thereby improving safety. We describe this lateral approach technique and review outcomes compared to the standard technique
\end{abstract}

Methods: From 2007 to 2019, we have conducted 13 annual surgical missions to low resource setting in Gitwe, Rwanda. Retrospective chart review of surgeries between 2012 and 2019 was performed to study outcomes using standard approach and lateral technique during the same time period.

Results: Over a period of 8 years (2012 to 2019), out of 192 total cases, lateral approach was used in 35 patients. No patient experienced significant intra-operative blood loss requiring transfusion. One patient had a post-operative hematoma requiring surgical intervention. Vocal cord mobility testing by transcutaneous laryngeal ultrasound was implemented in 2016. Of all patients, incidence of vocal cord weakness was $8.0 \%$ (11/137 patients tested) with less than $1 / 3$ of these symptomatic. There was no statistically significant difference in vocal cord weakness noted in the two approaches (3/23 in lateral approach and 8/114 in standard approach) by Fisher's exact test $(\mathrm{P}=0.34)$.

Conclusions: Lateral approach, by affording optimal exposure of the great vessels and the laryngeal nerves, reduces the risk of bleeding and nerve injury. Furthermore, inferiorly based strap muscle flap provides excellent coverage and cosmetic outcome.

Keywords: Backdoor; lateral approach; goiter; hypoparathyroidism; recurrent nerve injury; thyroidectomy; giant endemic goiter; transcutaneous laryngeal ultrasound (TCLUS)

Submitted Sep 22, 2020. Accepted for publication Dec 25, 2020.

doi: $10.21037 /$ gs-20-725

View this article at: http://dx.doi.org/10.21037/gs-20-725

\section{Introduction}

Surgical management of very large or mega-goiters seen in low resource endemic areas with extreme iodine deficiency presents unique challenges. Surgery of large goiters carries a significant risk of injury to recurrent laryngeal nerve (RLN) due to traction and potential of excessive blood loss resulting from inadequate exposure to the superior vascular pedicle (1). Surgery for this benign thyroid disease is justified if the complication rate is comparable to thyroid surgery done for other indications.

Based on our first 5-year experience (2007 to 2011) operating on mega-goiters in Gitwe, Rwanda, we developed 
an alternative approach to the superior pole that we have utilized in select cases for the last 8 years. We find our technique of lateral approach especially useful in large goiters with superior and lateral extension.

Some authors have recommended total thyroidectomy as the procedure of choice for bilateral benign multinodular goiter (2). However, we believe that total thyroidectomy may not be ideal as remnant of thyroid tissue may circumvent the need for postoperative thyroid hormone replacement which is an important consideration in the low resource settings of Sub-Saharan Africa. There are varied barriers to access for medication supplementation and follow up, especially in the remote areas. Moreover, partial or subtotal thyroidectomy eliminates the risk of airway compromise due to bilateral recurrent laryngeal injury and the need for monitoring of calcium levels. Therefore, we perform thyroid lobectomy for unilateral goiter and lobectomy with partial contralateral lobectomy (Dunhill procedure) for bilateral goiter. In our experience, the majority $(75 \%)$ of endemic goiters tend to be unilateral or predominantly involving one lobe. A significant number $(20 \%)$ of goiter patients have infraclavicular or substernal extension. A similar number have significant superior extension to the hyoid bone, and in some instances up to the mastoid process.

Superior extension of the goiter presents a unique problem putting at high risk the laryngeal nerves and the superior pole vessels. In some instances where the goiters are very large, lateral extension results in involvement of the carotid artery and the internal jugular vein which are often adherent to the thyroid capsule obliterating the natural surgical planes.

We aim to introduce a novel surgical technique and demonstrate its safety with regards to RLN injury, hypocalcemia, and blood loss. We present the following data in accordance with the STROBE reporting checklist (available at http://dx.doi.org/10.21037/gs-20-725).

\section{Methods}

We present a retrospective chart review of 192 patients who underwent thyroid lobectomy or subtotal thyroidectomy in Gitwe, Rwanda between 2012 and 2019 for mega goiters causing compressive symptoms. Patients underwent either traditional approach (157 patients) or lateral technique (35 patients, $18.2 \%)$. We describe selection criteria, surgical technique, and outcome measures utilizing this technique. All data is taken prior to discharge from the hospital. No consistent long-term follow-up is available, a common limitation of surgical mission trips. Data was taken from a de-identified database kept by the senior surgeons over the years of the surgical mission trips. Therefore, patient identifying variables including age and sex are not available. The study was conducted in accordance with the Declaration of Helsinki (as revised in 2013). The study did not require ethical board approval as the dataset had no patient identifiers. Patients endorsed care by signing written consent for thyroid surgery, which was translated verbally into Kinyarwandese by local translators if the patient did not communicate in English. This study is non-blinded, therefore clinical judgement and the retrospective nature of the study may introduce inherent bias.

\section{Statistical analysis}

Primary outcome measures were vocal cord paresis measured by transcutaneous laryngeal ultrasound (TCLUS) and blood loss requiring transfusion or return to the operating room. Statistical analysis was performed using Fisher's exact test.

\section{Selection criteria for lateral approach}

Patients with World Health Organization (WHO) stage 4 thyroid goiter, causing pressure symptoms are offered surgical intervention. All surgical candidates are assessed for their thyroid status utilizing thyroid stimulating hormone (TSH) levels. In addition to the physical exam, ultrasound is performed with close attention to superior extension of the gland noting location relative to the hyoid, inferior extension of the gland above or below the clavicle, and vascularity of the gland, specifically the superior pole vessels. Additionally, vocal cord movement is tested by TCLUS. Goiters with superior extension of the gland at or above the level of the hyoid or large caliber of the superior thyroid artery are chosen for lateral approach. Inferior extension of the gland below the clavicle did not qualify the patient for lateral approach. An example of a mega goiter chosen for lateral approach can be seen in Figure 1 .

\section{Operative technique}

The operative technique consists of standard skin crease incision. Subplatysmal flaps are raised. The fascia along the anterior border of the sternocleidomastoid muscle (SCM) is incised on the side of the goitrous lobe and the SCM is retracted laterally (Figure 2). The internal jugular vein 


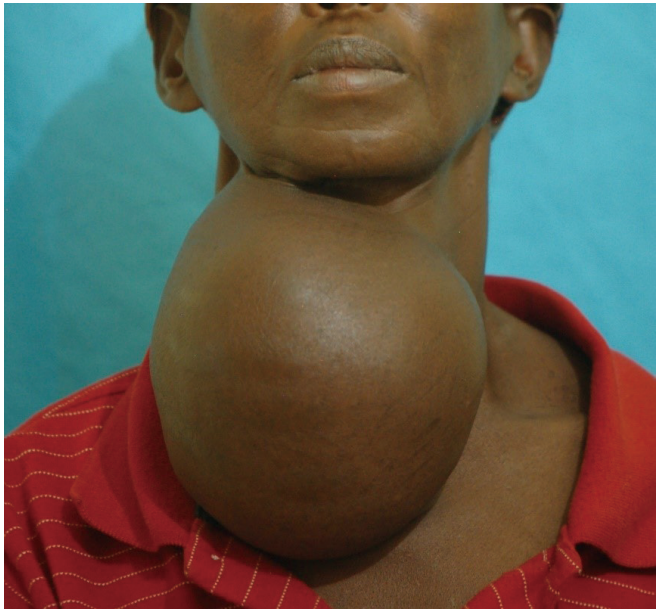

Figure 1 Example of mega goiter chosen for lateral approach.



Figure 2 Retraction of sternocleidomastoid muscle (SCM) laterally to expose great vessels.

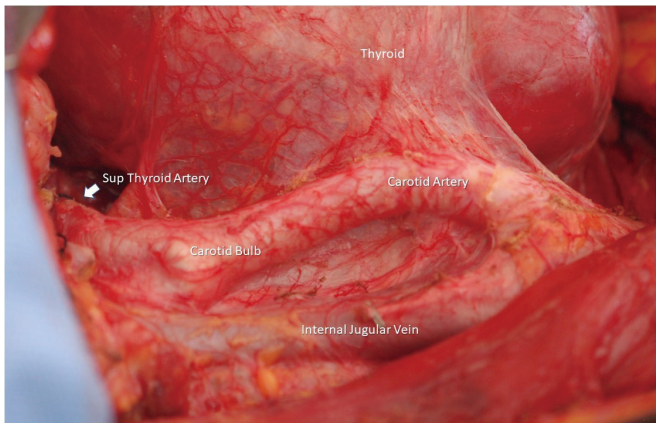

Figure 3 Great vessels seen abutting thyroid gland.

and carotid artery are exposed. In some cases, the great vessels are adherent to the goiter (Figure 3) and have to be carefully dissected. Lateral approach makes it possible to do this maneuver safely. The strap muscles fibers can be seen

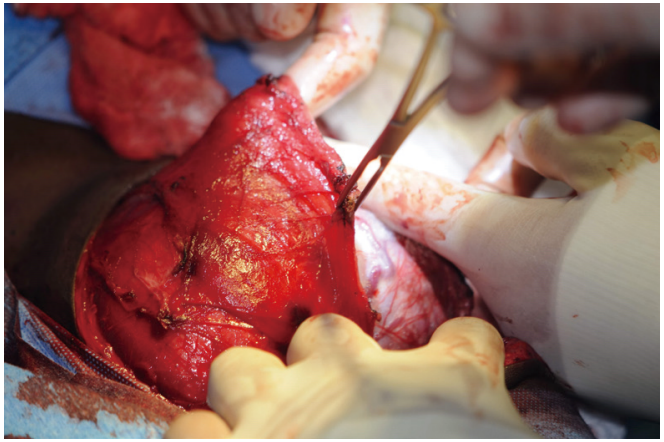

Figure 4 Strap muscles splaying goiter and beginning of muscular flap elevation.

splayed by the goiter (Figure 4). A horizontal incision is made through the sternohyoid and sternothyroid preserving a 2 -cm cuff of muscle at the superior attachment. The muscular flap is gently elevated off the capsule and reflected inferiorly, creating an inferiorly based strap muscle flap. The superior thyroid artery and vein are then exposed. Depending on the superior extent, the goiter is often dissected from the tail of the parotid or the submandibular gland. Hypoglossal nerve is dissected and preserved. This approach also offers an excellent view of the superior laryngeal nerve. Once all the nerve structures are identified, dissected, and preserved, attention is paid to the superior pole vessels-superior thyroid artery and vein, which are ligated and divided. Once the superior pole vasculature is divided, the goiter is retracted medially and the fascia or the capsule of the gland is entered medially and "high up" on the gland to preserve parathyroid glands and their vascular supply. Fascia is gently swept off the surface of the gland in a medial to lateral direction, making sure to avoid big sweeps while carefully looking for middle thyroid vein. The RLN and the inferior thyroid artery (ITA) are identified (Figure 5). Superior parathyroid gland is seen dorsal to the RLN. ITA is ligated distally preserving the blood supply to the superior parathyroid gland and the goiter can be reflected further medially. The isthmus is divided in case of lobectomy and gland removed from the trachea by dividing Berry's ligament. In cases of subtotal thyroidectomy, partial removal of the contralateral lobe is performed making sure to keep the dissection anterior to the trachea and leaving a healthy cuff of thyroid tissue laterally. The muscular flap is re-approximated to the superior remnant of strap musculature (Figure 6). A drain is placed, and the wound is closed in layers. 


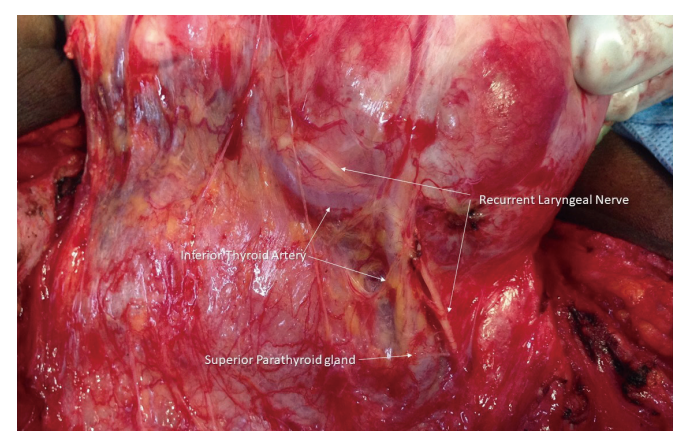

Figure 5 Demonstration of relationship of Inferior thyroid artery, recurrent laryngeal nerve and superior parathyroid gland.

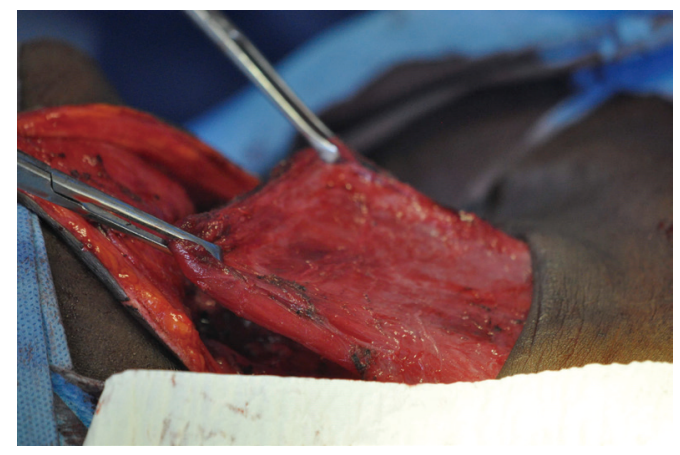

Figure 6 Re-approximation of muscular flap.

Table 1 Lateral approach cases by year

\begin{tabular}{lccc}
\hline Year & Lateral approach & Total cases & Cases by year \\
\hline 2012 & 2 & 19 & $10.53 \%$ \\
2013 & 3 & 18 & $16.67 \%$ \\
2014 & 3 & 20 & $15.00 \%$ \\
2015 & 4 & 21 & $19.05 \%$ \\
2016 & 5 & 26 & $19.23 \%$ \\
2017 & 6 & 32 & $18.75 \%$ \\
2018 & 6 & 27 & $22.22 \%$ \\
2019 & 6 & 29 & $20.69 \%$ \\
Total & 35 & 192 & $18.23 \%$ \\
\hline
\end{tabular}

\section{Results}

Based on the initial 5-year experience (2007-2011) of operating on mega goiters using the traditional approach, senior author developed the alternative lateral approach
Table 2 Transcutaneous laryngeal ultrasound

\begin{tabular}{lccc}
\hline Group & $\mathrm{N}^{\dagger}$ & $\begin{array}{c}\text { Post-op vocal cord } \\
\text { paresis, } \mathrm{n}^{\ddagger}(\%)\end{array}$ & $\begin{array}{c}\text { Symptomatic } \\
\text { paresis }\end{array}$ \\
\hline Lateral approach & 23 & $3(13.0)$ & $1(4.3)$ \\
Traditional approach & 114 & $8(7.0)$ & $2(1.8)$ \\
Total & 137 & $11(8.0)$ & $3(2.2)$ \\
\hline
\end{tabular}

${ }^{\dagger}, \mathrm{N}=$ total number of thyroid cases performed with either lateral or traditional approach since 2016. ${ }^{\ddagger}, \mathrm{n}=$ number of patients with vocal cord paresis by either lateral or traditional approach. ${ }^{\S}$, symptomatic paresis percentage taken from number of patients in either the lateral approach, traditional approach, or total TCLUS patients. TCLUS, transcutaneous laryngeal ultrasound.

for mega-goiters with significant superior extension, as they represent unique challenge due to difficult exposure. Since 2012 , a total of $35 / 192$ cases $(18.2 \%)$ have been done utilizing this approach. Number of cases done by this approach per year has slowly increased from $15 \%$ to $21 \%$ over the last 5 years (Table 1).

\section{Surgical outcome}

\section{Bleeding}

No patients experienced large enough intra-operative blood loss to require transfusion. One patient in the traditional approach had a post-operative hematoma requiring surgical intervention $(0.5 \%$ of total patients). All patients had adequate pain control with acetaminophen post-operatively.

\section{Vocal cord dysfunction}

Starting in 2016, we introduced pre-operative and postoperative TCLUS for vocal cord mobility assessment. Over a period of 4 years, we performed TCLUS in 137 patients (Table 2). Incidence of vocal cord weakness by TCLUS was $8.0 \%$ (11/137 patients tested) with less than $1 / 3$ of these symptomatic. There was no statistically significant difference in vocal cord weakness noted in the two approaches when analyzed by Fisher's exact test $(\mathrm{P}=0.34)$, however there was a trend towards greater vocal cord paresis in the lateral approach group.

\section{Hypocalcemia}

One patient in the lateral approach cohort had unexplained symptoms of paresthesia after thyroid lobectomy that improved with empiric oral calcium carbonate. Laboratory values were not available for measurement of hypocalcemia 
due to financial constraints.

\section{Discussion}

Surgical management of endemic goiters is challenging due to the high risk of bleeding and injury to recurrent laryngeal and other cranial nerves due to limited exposure. These surgeries are usually performed in a low resource setting and done for benign disease. It is important that the complication rate for performing these surgeries be kept at levels comparable to thyroid surgery done for other pathologies in well-equipped settings.

We have been performing these surgeries in low resource setting in Gitwe, Rwanda since 2007. As we gained experience operating on large goiters with superior extension, we realized limitations of the traditional approach in fully exposing the major blood vessels in the neck. In an effort to improve exposure, the neck incision was extended superiorly, but this proved inadequate. Additionally, we had a case with significant blood loss of more than $500 \mathrm{~mL}$ in a patient with an internal jugular vein venotomy. This led to the development of our lateral approach. We then developed selection criteria, refined the technique, and incorporated an inferiorly based muscle flap to achieve improved neurovascular protection and better cosmesis. Incorporation of TCLUS in our practice allowed us to further analyze this technique and measure surgical outcomes objectively.

This technique differs from the traditional thyroidectomy technique in several ways. After subplatysmal flaps are raised, instead of dividing the midline raphe as would be done in the traditional approach, exposure is first gained laterally similar to a neck dissection by opening the fascia enveloping the SCM and exposing the great vessels. This allows a plane to be safely developed between the great vessels and the gland which is often abutting the medial wall of the carotid or internal jugular vein. Additionally, the inferiorly based muscular flap distinguishes this technique from the traditional thyroidectomy approach and aids in dissection of the superior pole vessels.

Current review of the technique demonstrates that the lateral technique is beneficial in vascular control intraoperatively and may reduce risk of the RLN injury in surgery of large goiters. We had no major intra-operative blood loss and one patient in our non-lateral (traditional) approach cohort had a post-operative hematoma requiring surgical intervention ( $0.5 \%$ total patients). A recent metaanalysis described a range of reported post-thyroidectomy hematomas requiring surgical intervention from $0.4 \%$ to $6.5 \%$ (3). Risk factors for hematoma include male gender, age, Graves' disease, hypertension, antithrombotic drug use, thyroid procedure in low-volume hospitals, prior thyroid surgery, total thyroidectomy, and neck dissection (3). Our patients do not belong to these high-risk categories, but we believe they present a similar or higher risk of bleeding due to the size and vascularity of these glands. We attribute our low incidence of bleeding in our series to improved exposure of the vascular pedicle allowing for better control.

In our study, 11 out of 137 (8.0\%) patients with available TCLUS data had post-operative vocal cord paresis, with less than a third of these patients with dysphonia. Although the difference in vocal cord paresis was not significantly different between the two approaches, the lateral approach group had a higher incidence of vocal cord paresis. This is likely due to the patient population that was selected for lateral approach having larger and more superior glands necessitating more extensive dissection which was safely done through our lateral approach. No patients had swallowing difficulties or choking symptoms. Recent literature has shown a transient vocal cord palsy rate of $6-10.6 \%$ and permanent vocal cord palsy rate of $0.6-2.3 \%$ in thyroidectomy \& parathyroidectomy patients (4-6). We do not have long-term laryngeal ultrasound findings to assess for permanent vocal cord palsy. We believe that low incidence of vocal cord palsy in our cohort is due to excellent exposure afforded by the lateral approach.

Previous authors have described a lateral approach without the use of muscular flaps $(7,8)$. Dissanayake et al. described the use of lateral approach without muscular flaps to be especially useful for reoperations (8). El-Erian et al. described a technique that involved accessing the superior pole through the carotid triangle (7). We find that very superior goiters often distort the anatomy as the gland may occupy part of the carotid triangle and traditional anatomic relationships may be unreliable. Ko et al. described a U-shaped muscular flap to aid in operative exposure that is similar to our muscular flap, but their approach does not place an emphasis on opening the investing fascia of the sternocleidomastoid and exposing the great vessels prior to dissection of the thyroid gland (9).

Some have argued that preserving strap musculature is beneficial for swallowing and voice (7). Long-term impact of dividing the strap musculature is debatable. Henry et al. found no short- or long-term effects on voice in patients with sternothyroid muscle division (10). It is well described in the literature that dysphagia may occur after 
thyroidectomy but typically resolves within 3 months (11). Strap muscle division has not been shown to definitively worsen swallowing function after thyroidectomy. We believe that improved exposure and thereby greater safety is a fair trade off for possible minor alterations in voice or swallowing due to division of strap musculature. These swallowing difficulties, if present, are likely to be temporary as the strap muscle flap is meticulously re-approximated at the end of the procedure.

Our criteria for choosing the lateral approach are based on the extent of superior extension of the goiter above the hyoid bone and enlarged superior vascular pedicle by ultrasound. Infraclavicular extension of the gland did not qualify the patient for lateral approach, but was noted on preoperative ultrasound to aid in operative planning. The lateral approach is not beneficial for goiters with infraclavicular extension without suprahyoid extension or large superior pole vessels. The most useful aspect of this lateral approach is the control of superior pole vessels, which additionally affords identification of the external branch of the superior laryngeal nerve. Having access to the external carotid artery and internal jugular vein affords additional confidence and expediency in case of inadvertent venotomy/arteriotomy in dissection of the engorged superior pedicle vessels. Once the superior pedicle is under control, it becomes easier to rotate the gland and get exposure and dissection of the RLN. Additionally, the muscular flap provides adequate post-operative cosmesis and symmetry. Our technique may prove useful in difficult thyroid cancer surgeries wherein lateral exposure of the great vessels and a muscular flap can aid in safe dissection of large thyroid tumors or revision cases. Our described technique leaves a remnant thyroid tissue of the nongoitrous lobe, which would be contraindicated in advanced thyroid cancers. To modify this technique for thyroid cancer surgery, bilateral incision of investing fascia of sternocleidomastoid and exposure of bilateral great vessels is necessary. Additionally, instead of performing the Dunhill procedure, a total thyroidectomy would be performed.

Limitations of this study include lack of long-term follow-up of vocal cord mobility in patients with vocal cord weakness, retrospective nature of the study, and lack of calcium lab data due to scarce laboratory resources.

\section{Conclusions}

This study introduces a novel technique for surgical management of large goiters with superior extension. We believe that this alternative approach is a useful tool not only in the low resource settings for management of megagoiters, but should be a consideration in difficult thyroid cancer cases, especially those with bulky lateral neck lymphadenopathy and in revision surgeries.

\section{Acknowledgments}

The surgical mission trips received financial and resource assistance through Medical Missions for Children. This work was presented as a virtual poster at the Combined Otolaryngology Spring Meeting in April 2020.

Funding: None.

\section{Footnote}

Reporting Checklist: The authors have completed the STROBE reporting checklist. Available at http://dx.doi. org/10.21037/gs-20-725

Data Sharing Statement: Available at http://dx.doi. org/10.21037/gs-20-725

Peer Review File: Available at http://dx.doi.org/10.21037/gs20-725

Conflicts of Interest: All authors have completed the ICMJE uniform disclosure form (available at http://dx.doi. org/10.21037/gs-20-725). The authors have no conflicts of interest to declare

Ethical Statement: The authors are accountable for all aspects of the work in ensuring that questions related to the accuracy or integrity of any part of the work are appropriately investigated and resolved. The study was conducted in accordance with the Declaration of Helsinki (as revised in 2013). The study did not require ethical board approval as the dataset had no patient identifiers. Patients endorsed care by signing written consent for thyroid surgery, which was translated verbally into Kinyarwandese by local translators if the patient did not communicate in English.

Open Access Statement: This is an Open Access article distributed in accordance with the Creative Commons Attribution-NonCommercial-NoDerivs 4.0 International License (CC BY-NC-ND 4.0), which permits the noncommercial replication and distribution of the article with 
the strict proviso that no changes or edits are made and the original work is properly cited (including links to both the formal publication through the relevant DOI and the license). See: https://creativecommons.org/licenses/by-nc-nd/4.0/.

\section{References}

1. Ríos A, Rodríguez JM, Canteras M, et al. Surgical management of multinodular goiter with compression symptoms. Arch Surg 2005;140:49-53.

2. Agarwal G, Aggarwal V. Is total thyroidectomy the surgical procedure of choice for benign multinodular goiter? An evidence-based review. World J Surg 2008;32:1313-24.

3. Fan C, Zhou X, Su G, et al. Risk factors for neck hematoma requiring surgical re-intervention after thyroidectomy: a systematic review and meta-analysis. BMC Surg 2019;19:98.

4. Jeannon JP, Orabi AA, Bruch GA, et al. Diagnosis of recurrent laryngeal nerve palsy after thyroidectomy: a systematic review. Int J Clin Pract 2009;63:624-9.

5. Joliat GR, Guarnero V, Demartines N, et al. Recurrent laryngeal nerve injury after thyroid and parathyroid surgery: Incidence and postoperative evolution assessment.
Medicine (Baltimore) 2017;96:e6674.

6. Meltzer C, Hull M, Sundang A, et al. Association Between Annual Surgeon Total Thyroidectomy Volume and Transient and Permanent Complications. JAMA Otolaryngol Head Neck Surg 2019. [Epub ahead of print]. doi: 10.1001/jamaoto.2019.1752.

7. El-Erian AM, El-Raouf AA, Nabeel I, et al. Lateral Approach to Attack Superior Thyroid Vascular Pedicle Eliminates the Need for Strap Muscles Cutting during Thyroidectomy. Medical Journal of Cairo University 2015;83:125-34.

8. Dissanayake DDMC, Fernando RF, Dissanayake IL. Lateral approach to Thyroid: A Good Technique for Reoperative Thyroid Surgery. World Journal of Endocrine Surgery 2016;8:141-2.

9. Ko HY, Lu IC, Chang PY, et al. U-shaped strap muscle flap for difficult thyroid surgery. Gland Surg 2020;9:372-9.

10. Henry LR, Solomon NP, Howard R, et al. The functional impact on voice of sternothyroid muscle division during thyroidectomy. Ann Surg Oncol 2008;15:2027-33.

11. Lombardi CP, Raffaelli M, De Crea C, et al. Long-term outcome of functional post-thyroidectomy voice and swallowing symptoms. Surgery 2009;146:1174-81.
Cite this article as: DeBiase C, Sebelik M, Chandra SR, Dhingra J. Lateral approach improves surgical access to the superior pole in giant endemic goiters. Gland Surg 2021;10(3):973-979. doi: 10.21037/gs-20-725 